\title{
CHEMICAL AND BIOMATERIALS ASPECTS OF CALCIUM PHOSPHATE CEMENT
}

\author{
Hideki Monma* \\ (*Corresponding author:monma@cc.kogakuin.ac.jp) \\ Department of Materials Science and Technology, \\ Faculty of Engineering, Kogakuin University \\ 2665 Nakano-cho, Hachioji-shi, Tokyo 192-0015
}

Keywords: Hydroxyapatite, Calcium phosphate cement, Bone paste, Porous ceramics, Scaffold

Calcium phosphate cements and the resulting porous hardened bodies are noticed as injectable bone pastes and scaffolds for bone ingrowth or bone cell culture, respectively. The present paper provides various calcium phosphate cement combinations, chemistry and hardened properties.

(Received May 5, 2006; Accepted December 15, 2006)

\section{INTRODUCTION}

Materials in the systems $\mathrm{CaO}-\mathrm{Al}_{2} \mathrm{O}_{3}$, $\mathrm{CaO}-\mathrm{SiO}_{2}$ and $\mathrm{CaO}-\mathrm{SO}_{3}$ are well-known as typical cement constituents, i.e., the first two systems are for Portland cement and the third one for gypsum. In 1970's, hydroxyapatite (referred to as HAp, stoichiometrically $\mathrm{Ca}_{10}\left(\mathrm{PO}_{4}\right)_{6} \quad(\mathrm{OH})_{2}$, nonstoichiometrically $\mathrm{Ca}_{10-x}$ (HP

$\left.\left.\mathrm{O}_{4}\right)_{x}\left(\mathrm{PO}_{4}\right)_{6-x}(\mathrm{OH})_{2-x} \quad n \mathrm{H}_{2} \mathrm{O}\right)$ had been noticed as a future promising biomaterial for bone and tooth restorations, and many preparative and materials researches on HAp have been continued. And in 1976, a unique hydration and hardening property of $\alpha-\mathrm{Ca}_{3}\left(\mathrm{PO}_{4}\right)_{2}$ powder was found ${ }^{1)}$. Nearly the same time a $\mathrm{Ca}_{4}\left(\mathrm{PO}_{4}\right)_{2} \mathrm{O}-\mathrm{CaHPO}_{4} \square 2 \mathrm{H}_{2} \mathrm{O}$ mixed type cement was developed ${ }^{2)}$. Each of the two cementing calcium phosphates (CP cements) was a pioneer for succeeding developments of various CP cements. Presently some CP cements are already applied to the medical treatment of bone and tooth. On the other hand, recently porous calcium phosphate ceramics have become of interest as a structural morphology with good bioactivity, i.e., scaffolds for bone ingrowth or for bone cell culture. Such porous ceramics could be easily prepared by using CP cements. In the present paper, CP cements are introduced from chemical and biomaterial aspects.

\section{CP CEMENTS}

Chemistry of CP cement

$\mathrm{CP}$ cements harden with the formation of mainly HAp by mixing with aqueous solution. Many types of CP cements have been created by various combinations of components. And properties of CP cements have been examined with respect to hardening mechanism, hardened microstructure, mechanical strength and so forth. Fig.1 shows examples of $\mathrm{CP}$ cement hardened bodies and sintered ceramics prepared by using CP cement. Such porous materials have an excellent 
biocompatibility, and are expected as craffolds for hone incrowith

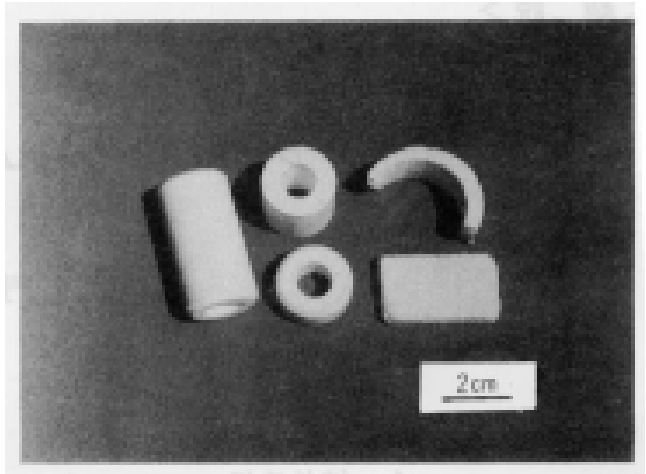

Fig.1 HAp porous blocks prepared by using $\alpha$ TCP cement. Left 3 blocks : Hydration-hardening bodies, Right 2 blocks: Ceramics obtained by sintering hydration-hardening bodies.

and bone cell culture. The hydration-hardening of CP cements occurs according to the dissolution-precipitation mechanism, i.e., by the entanglement of formed microcrystals such as HAp, as well as the hardening mechanism of gypsum hemihydrate $\mathrm{CaSO}_{4} \square 0.5 \mathrm{H}_{2} \mathrm{O}$. $\mathrm{HAp}$ is the most stable phase in a wide $\mathrm{pH}$ region ${ }^{3)}$ as shown in Fig. 2. Possibilities of the hydration-hardening of CP cement compositions could be predicted by comparing the solubility of calcium phosphates, which were already discussed in detail in the literature ${ }^{4)}$. In other words, all compounds being over the solubility curve of HAp tend to change into HAp.

The cement hardening reaction must proceed without stirring and with approximate rates for the entanglement of formed HAp microcrystals. For example, the change of acidic $\mathrm{CaHPO}_{4} \square 2 \mathrm{H}_{2} \mathrm{O}$ into basic HAp causes $\mathrm{pH}$ decreases with the reaction, therefore the reaction must stop at the point A (e.g., singular point) in Fig.
2. In the case of $\mathrm{Ca}_{4}\left(\mathrm{PO}_{4}\right)_{2} \mathrm{O}-\mathrm{CaHPO}_{4} \mathrm{C}$ $2 \mathrm{H}_{2} \mathrm{O}$ mixed type cement ${ }^{2)}$, solubility

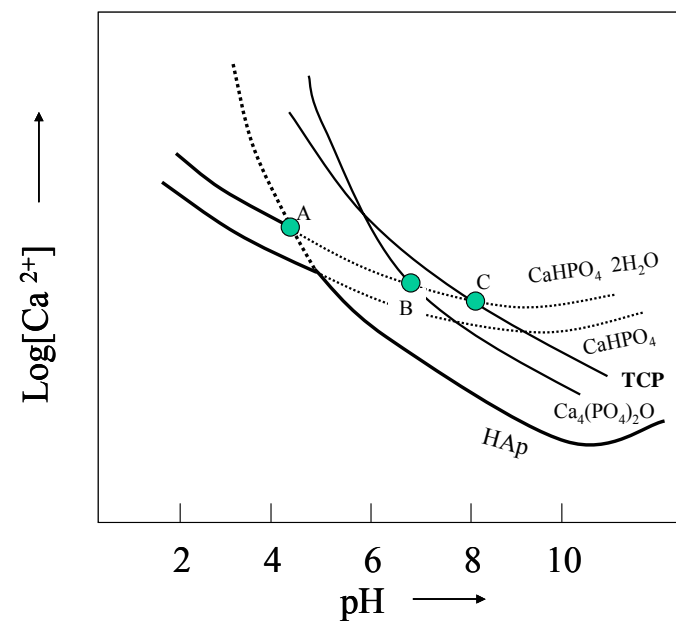

Fig.2 Solubility curves of calcium phosphates with respect to $\mathrm{pH}$ $(\text { calculated })^{3)}$.

curves of the two components are crossing at $\mathrm{pH} 6.8$ (point $\mathrm{B}$ ), and the point is above the solubility of HAp. So, when the $\mathrm{pH}$ of the two component system shifts from the point, $\mathrm{Ca}_{4}\left(\mathrm{PO}_{4}\right)_{2} \mathrm{O}$ or $\mathrm{CaHPO}_{4} \square 2 \mathrm{H}_{2} \mathrm{O}$ dissolves for maintaining the singular point until either of $\mathrm{Ca}_{4}\left(\mathrm{PO}_{4}\right)_{2} \mathrm{O}$ or $\mathrm{CaHPO}_{4} \mathrm{C}$ $2 \mathrm{H}_{2} \mathrm{O}$ disappears. Under such a thermodynamic consideration, this mixed type cement was developed ${ }^{2)}$. Similarly the $\mathrm{pH}$ of the system $\alpha$ TCP- $\mathrm{CaHPO}_{4} \square 2 \mathrm{H}_{2} \mathrm{O}$ must be automatically kept at 8 (point $\mathrm{C}$ ). And the hardening was experimentally confirmed $^{5)}$.

$\mathrm{CaHPO}_{4} \longrightarrow 2 \mathrm{H}_{2} \mathrm{O}-\mathrm{CaCO}_{3}$ and $\beta \mathrm{TCP}-$ $\mathrm{Ca}\left(\mathrm{H}_{2} \mathrm{PO}_{4}\right) \square \mathrm{H}_{2} \mathrm{O}$ systems have also been developed. Table 1 summarized various $\mathrm{CP}$ cement compositions and hardened properties. At present medically applied CP cements are two types consisting of $\alpha \mathrm{TCP}$ and $\mathrm{Ca}_{4}\left(\mathrm{PO}_{4}\right)_{2} \mathrm{O}$ as the main constituent. Only $\alpha$ TCP hardens without any other additives. Other $\mathrm{CP}$ cements are made by combinations of at least two components. 
$\alpha$ TCP-based cements ${ }^{1)}$

Chemical reactivity of the high tempera

Table 1 CP cement compositions and their hardened properties ${ }^{6)}$.

\begin{tabular}{|l|l|l|l|}
\hline $\begin{array}{l}\text { Main- } \\
\text { component }\end{array}$ & $\begin{array}{l}\text { Combinated- } \\
\text { components }\end{array}$ & $\begin{array}{l}\text { Hydration-hardened body } \\
\text { Hardening time, } t / \mathrm{min} \\
\text { porosity, } p / \% \\
\text { Compressive, } \sigma / \mathrm{MPa}\end{array}$ & $\begin{array}{l}\text { Organic acid-hardened } \\
\text { body } \\
\text { Hardening time, } t / \mathrm{min} \\
\text { Porosity, } p / \% \\
\text { Compressive, } \sigma / \mathrm{MPa}\end{array}$ \\
\hline$\alpha \mathrm{TCP}$ & None & $t=4-30, p=55-80, \sigma=3-18^{\mathrm{b})}$ & $t=2-8, \sigma=20-150$ \\
\hline$\alpha \mathrm{TCP}$ & $\mathrm{CaHPO}_{4} \square 2 \mathrm{H}_{2} \mathrm{O}$ & $t=9-10, p=48-56, \sigma=14-19$ & \\
\hline$\alpha \mathrm{TCP}$ & $\mathrm{CaHPO}_{4}$ & $t=c a .100$ & \\
\hline $\mathrm{Ca}_{4}\left(\mathrm{PO}_{4}\right)_{2} \mathrm{O}$ & $\mathrm{None}$ & & $t=2-8, \sigma=40-110$ \\
\hline $\mathrm{Ca}_{4}\left(\mathrm{PO}_{4}\right)_{2} \mathrm{O}$ & $\mathrm{CaHPO}_{4} \square 2 \mathrm{H}_{2} \mathrm{O}$ & $t=c a .9$ & \\
\hline $\mathrm{Ca}_{4}\left(\mathrm{PO}_{4}\right)_{2} \mathrm{O}$ & $\mathrm{CaHPO}_{4}$ & $t=5-7, \sigma=5-9$ & \\
\hline $\mathrm{Ca}_{4}\left(\mathrm{PO}_{4}\right)_{2} \mathrm{O}$ & $\mathrm{CaHPO}_{4} \square 2 \mathrm{H}_{2} \mathrm{O}, \mathrm{HAp}$ & $t=7-8, \sigma=19-40$ & $t=2-20, \sigma=30-150$ \\
\hline $\mathrm{Ca}_{4}\left(\mathrm{PO}_{4}\right)_{2} \mathrm{O}$ & $\left.\alpha \mathrm{TCP}^{c}\right)$ & $t=c a .130, \sigma=2-20$ & \\
\hline $\mathrm{CaHPO}_{4} \square 2 \mathrm{H}_{2} \mathrm{O}$ & $\mathrm{CaCO}_{3}$ & $t=60-120, \mathrm{p}=64-68, \sigma=2-3$ & \\
\hline
\end{tabular}

a) Polycarboxylic acids, lactic polymer, citric acid, malic acid, etc.

b) Compressive strength of $80^{\circ} \mathrm{C}$-hardened bodies : 3-30 $\mathrm{MPa}$

c) Dilute $\mathrm{H}_{3} \mathrm{PO}_{4}$ solution used as mixing solution

ture $\alpha$-form of TCP is larger than thelow temperature $\beta$ TCP. This fact is reasonable since the $\alpha$-form must be thermodynamically metastable at room temperature. Reactions of $\alpha \mathrm{TCP}$ with $\mathrm{H}_{2} \mathrm{O}$ are summarized as follows depending on $\mathrm{pH}$,

In acidic region :

$$
\begin{aligned}
& \mathrm{Ca}_{3}\left(\mathrm{PO}_{4}\right)_{2}+\mathrm{H}_{2} \mathrm{O} \\
& \rightarrow 2 \mathrm{CaHPO}_{4} \square 2 \mathrm{H}_{2} \mathrm{O}+\mathrm{Ca}(\mathrm{OH})_{2}
\end{aligned}
$$

In neutral region :

$$
\begin{aligned}
& 3 \mathrm{Ca}_{3}\left(\mathrm{PO}_{4}\right)_{2}+7 \mathrm{H}_{2} \mathrm{O} \\
& \rightarrow \mathrm{Ca}_{8} \mathrm{H}_{2}\left(\mathrm{PO}_{4}\right)_{6} \square 5 \mathrm{H}_{2} \mathrm{O}+\mathrm{Ca}(\mathrm{OH})_{2}
\end{aligned}
$$

In basic region :

$$
\begin{gathered}
(1-x) \mathrm{Ca}_{3}\left(\mathrm{PO}_{4}\right)_{2}+3(2+n-x) \mathrm{H}_{2} \mathrm{O} \\
\rightarrow 3 \mathrm{Ca}_{10-x}\left(\mathrm{HPO}_{4}\right)_{x}\left(\mathrm{PO}_{4}\right)_{6-x}(\mathrm{OH})_{2-x} \\
\quad \square n \mathrm{H}_{2} \mathrm{O}+2(1-x) \mathrm{H}_{3} \mathrm{PO}_{4}
\end{gathered}
$$

where the product in Reaction (3) is a general formula $(x=0-1)$ of HAp. Hardening is observed according to Reactions (2) and (3). Reaction (1) might be too fast to harden due to the entanglements of produced particles with each other. The resulting hydration-hardening bodies are porous materials with $55-80 \%$ in porosity , below $20 \mu \mathrm{m}$ in pore size, and nonstoichiometric compositions near biological HAp $\quad 0<x<1$, $1.50<\mathrm{Ca} / \mathrm{P}<1.67)$. That is, the products are closed to biological apatite rather than so far developed stoichiometric $\mathrm{HAp}(\mathrm{Ca} / \mathrm{P}=$ 1.67) biomaterials. The hydration-hardening is accelerated or retarded by additives in the following $\operatorname{order}^{7,8)}$;

$$
\begin{aligned}
\mathrm{NH}_{4} & \text { salts }>\mathrm{Na} \text { salts }>\text { hone ) } \\
& >\mathrm{Ca} \text { salts } \gg \mathrm{Mg} \text { salts }
\end{aligned}
$$

The $\alpha$ TCP single component cement was a base of the first medical application to the tooth restoration in Japan in 1986. Fig. 3 shows mechanical strength $v s$, porosity plots for hardened bodies prepared from $\alpha$ TCP single component cement, compared with data on HAp sintered ceramics.

$\alpha \mathrm{TCP}-2 \mathrm{CaHPO}_{4}, 2 \mathrm{H}_{2} \mathrm{O}$ mixed type 
cement $^{5)}$ hardens with the formation of octacalcium phosphate resembling compositionally and structurally HAp. This combination has comparatively good hardening properties, e.g., $10 \mathrm{~min}$ in $37^{\circ} \mathrm{C}$-setting time.. The hardening occurs as a result of the following continuous reaction kept automatically at the crossing point $\mathrm{C}(\mathrm{ca} . \mathrm{pH} 8)$ as shown in Fig. 2.

$$
\begin{gathered}
2 \mathrm{Ca}_{3}\left(\mathrm{PO}_{4}\right)_{2}+2 \mathrm{CaHPO}_{4} \square 2 \mathrm{H}_{2} \mathrm{O}+\mathrm{H}_{2} \mathrm{O} \\
\rightarrow \mathrm{Ca}_{8} \mathrm{H}_{2}\left(\mathrm{PO}_{4}\right)_{6} \square 5 \mathrm{H}_{2} \mathrm{O}
\end{gathered}
$$

The reaction rate is larger than that of aTCP single component cement. Wet compressive strengths of thus obtained bodies were $c a$. $20 \mathrm{MPa}$ at the beginning and finally 50-60 MPa. Nowadays 70-90 MPa values have been achieved, and the cement is an recent topic as injectable bone and medically applied. pastes $^{10-12}$ ). Table 2 lists medical CP cement bone pastes developed so far

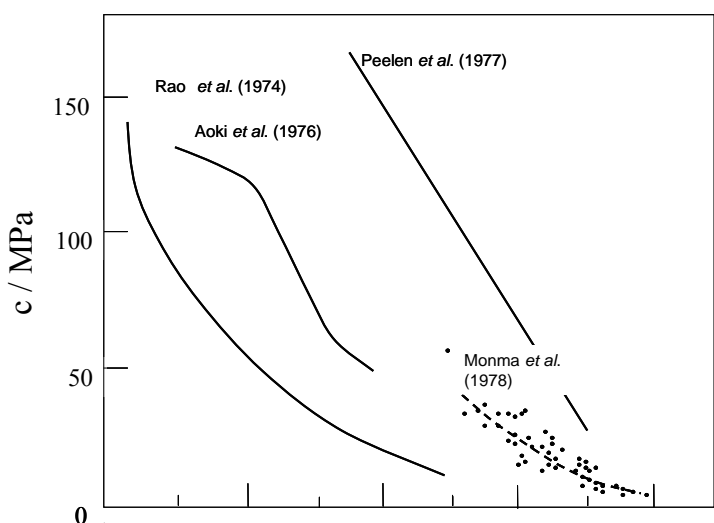

Fig. $3^{0}$ Relati20nships between 60 ompres $\$ 9 v e$ strength and porosity/\%of HAp porous bodies ${ }^{9}$. (dots : $\alpha$ TCP cement hardened bodies, solid lines : HAp ceramics)

\begin{tabular}{|c|c|c|c|c|c|}
\hline $\begin{array}{l}\text { Main- } \\
\text { component }\end{array}$ & $\begin{array}{l}\text { Combinated- } \\
\text { components }\end{array}$ & $\begin{array}{l}\text { Hydration-h } \\
\text { ardened } \\
\text { body }^{a)}\end{array}$ & $\begin{array}{l}\text { Final product } \\
\text { Features }\end{array}$ & $\begin{array}{l}\text { Commercial } \\
\text { name }\end{array}$ & Maker \\
\hline$\alpha \mathrm{TCP}$ & $\begin{array}{l}\mathrm{Ca}_{4}\left(\mathrm{PO}_{4}\right)_{2} \mathrm{O}, \\
\mathrm{CaHPO}_{4}\left[2 \mathrm{H}_{2} \mathrm{O},\right. \\
\mathrm{HAp}\end{array}$ & $\begin{array}{l}t=6-10 \\
\sigma=60-90\end{array}$ & $\begin{array}{l}\text { HAp } \\
\text { Bio-resorbable } \\
\text { High strength }\end{array}$ & Biopex & $\begin{array}{l}\text { Pentax } \\
\text { (Japan) }\end{array}$ \\
\hline$\alpha \mathrm{TCP}$ & $\begin{array}{l}\mathrm{CaCO}_{3}, \\
\mathrm{Ca}\left(\mathrm{H}_{2} \mathrm{PO}_{4}\right)_{2}\end{array}$ & $\begin{array}{l}t=8-9 \\
\sigma=28-38\end{array}$ & $\begin{array}{l}\text { HAp } \\
\text { Bio-resorbable } \\
\text { High initial } \\
\text { strength }\end{array}$ & Norian SRS & $\begin{array}{l}\text { Norian } \\
\text { (USA) }\end{array}$ \\
\hline$\alpha \mathrm{TCP}$ & $\begin{array}{l}\mathrm{Ca}_{4}\left(\mathrm{PO}_{4}\right)_{2} \mathrm{O}, \\
\mathrm{Ca}\left(\mathrm{H}_{2} \mathrm{PO}_{4}\right)_{2}\end{array}$ & $\begin{aligned} t & =16-18 \\
\sigma & =6-10\end{aligned}$ & $\begin{array}{l}\text { HAp } \\
\text { Low viscosity } \\
\text { paste }\end{array}$ & Cementek & $\begin{array}{l}\text { Teknimed } \\
\text { (France) }\end{array}$ \\
\hline$\alpha \mathrm{TCP}$ & $\begin{array}{l}\mathrm{Ca}_{4}\left(\mathrm{PO}_{4}\right)_{2} \mathrm{O}, \\
\mathrm{CaHPO}_{4}, \\
\mathrm{CaCO}_{3}, \mathrm{HAp}\end{array}$ & $\begin{aligned} t & =6-7 \\
\sigma & =79-87\end{aligned}$ & HAp & Biocement & $\begin{array}{l}\text { Merk } \\
\text { GmbH } \\
\text { (German) }\end{array}$ \\
\hline $\mathrm{Ca}_{4}\left(\mathrm{PO}_{4}\right)_{2} \mathrm{O}$ & $\mathrm{CaHPO}_{4}$, & & $\begin{array}{l}\text { HAp } \\
\text { Little bio- } \\
\text { resorbable }\end{array}$ & BoneSource & $\begin{array}{l}\text { Libinger } \\
\text { (USA) }\end{array}$ \\
\hline $\mathrm{Ca}_{4}\left(\mathrm{PO}_{4}\right)_{2} \mathrm{O}$ & $\mathrm{CaHPO}_{4}$, & $\begin{array}{l}t=5-20 \\
\sigma=50\end{array}$ & HAp & Cerapaste & $\begin{array}{l}\text { NGK } \\
\text { (Japan) }\end{array}$ \\
\hline $\mathrm{ACP}^{\mathrm{b})}$ & $\mathrm{CaHPO}_{4} \square 2 \mathrm{H}_{2} \mathrm{O}$ & $\begin{array}{l}t=18-20 \\
\sigma=3-5\end{array}$ & HAp & $\alpha-\mathrm{BSM}$ & $\begin{array}{l}\text { ETEX } \\
\text { (USA) }\end{array}$ \\
\hline
\end{tabular}

Table 2 CP cement bone paste developed ${ }^{12)}$.

a) $t$ and $\sigma$ mean hardening time (min) and compressive strength (MPa). 
b) Amorphous calcium phosphate, $\mathrm{Ca}_{3}\left(\mathrm{PO}_{4}\right)_{2} \square n \mathrm{H}_{2} \mathrm{O}$

$$
+3 x \mathrm{Ca}_{3}\left(\mathrm{PO}_{4}\right)_{2}
$$

\author{
$\mathrm{Ca}_{4}\left(\mathrm{PO}_{4}\right)_{2} \mathrm{O}$-based cements ${ }^{2,4)}$ \\ and others ${ }^{13)}$ \\ $\mathrm{CaHPO}_{4}$ or $\left.\mathrm{CaHPO}_{4}\right) \square 2 \mathrm{H}_{2} \mathrm{O}$ as a \\ combinated component and $\mathrm{HAp}$ seed \\ crystals are mixed, and dilute $\mathrm{H}_{3} \mathrm{PO}_{4}$ \\ solution is used as a mixing liquid. The \\ reaction is given as
}

$$
\begin{gathered}
2 \mathrm{Ca}_{4}\left(\mathrm{PO}_{4}\right)_{2} \mathrm{O}+2 \mathrm{CaHPO}_{4} \square 2 \mathrm{H}_{2} \mathrm{O}+\mathrm{H}_{2} \mathrm{O} \rightarrow \\
\mathrm{Ca}_{10}\left(\mathrm{PO}_{4}\right)_{6}(\mathrm{OH})_{2}+4 \mathrm{H}_{2} \mathrm{O}
\end{gathered}
$$

Many other cement combinations have been studied, e.g., $\mathrm{CaHPO}_{4} \square 2 \mathrm{H}_{2} \mathrm{O}-\mathrm{CaCO}_{3}$ system $37^{\circ} \mathrm{C}$-hardening time:1-2 $\mathrm{h}$, wet compressive : ca. $1 \mathrm{MPa}$,porosity:70- 80\%, product : carbonated HAp ) , $\beta \mathrm{TCP}-$ $\mathrm{Ca}\left(\mathrm{H}_{2} \mathrm{PO}_{4}\right)_{2} \square \mathrm{H}_{2} \mathrm{O}$ system, and cement compositions above over 400 varieties.

\section{HAp porous bodies prepared}

by using CP cement

Recently calcium phosphate porous ceramics have become of great interest as an excellent scaffold structure for osteoblast culture and bone ingrowth. $\mathrm{CP}$ cements are available for producing such porous materials ${ }^{14)}$. Increasing the hydration temperature increases the mechanical strength of hardened bodies. The $80^{\circ} \mathrm{C}$-hydrated HAp hardened bodies are machinable. By heating the products crack-free sintered apatite ceramics are prepared. During the heat-treatment, the following thermal change occurs over $700-800^{\circ} \mathrm{C}$.

$$
\begin{aligned}
& \mathrm{Ca}_{10-x}\left(\mathrm{HPO}_{4}\right)_{x}\left(\mathrm{PO}_{4}\right)_{6-x}(\mathrm{OH})_{2-\mathrm{x}} \square n \mathrm{H}_{2} \mathrm{O} \\
& \quad \rightarrow(1-x) \mathrm{Ca}_{10}\left(\mathrm{PO}_{4}\right)_{6}(\mathrm{OH})_{2}
\end{aligned}
$$

Therefore, the produced ceramics are composed of HAp single phase or HAp+TCP mixed phases or $\beta$ TCP single phase depending on the degree $(x)$ of the nonstoichiometry of reactant HAp. When the $\mathrm{Ca} / \mathrm{P}$ molar ratio of the $\mathrm{CP}$ cement used could be adjusted with the addition of appropriate calcium salts, HAp single phase ceramics with $4-20 \%$ in porosity, 2-5 $\mu \mathrm{m}$ in pore diameter and 100-500 $\mathrm{MPa}$ in compressive strength are prepared. By using polymer beads porous HAp with $20-500 \mu \mathrm{m}$ in pore size and $30-65 \%$ in porosity could be easily prepared. By using aTCP cement with albumin as a pore forming reagent, HAp bodies with large pores of $100-500 \mu \mathrm{m}$ are prepared ${ }^{15)}$. Figs.4-6 show microstructures of HAp porous materials prepared by using hydration reactions. These porous materials are applicable not only to medical engineering materials but also to catalyst support, ion-exchanger, adsorbent of smelling gasses, drag delivery system (DDS) carrier and so forth.

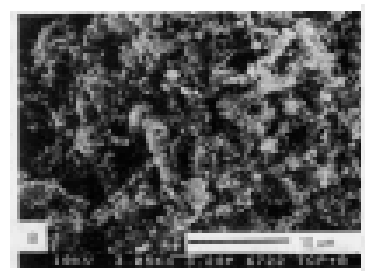

A

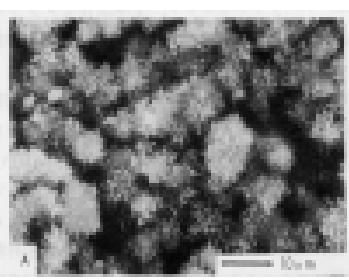

B
Fig. 4 SEM photographs of HAp porous materials. A: $\alpha \mathrm{TCP}$ cement r.t.-hardened body, B: $\alpha \mathrm{TCP}$ cement $80^{\circ} \mathrm{C}$-hardened body,
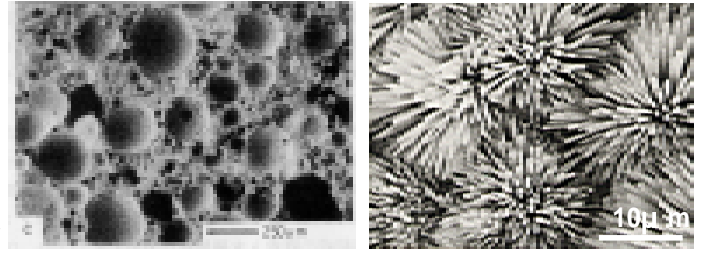


\section{REFERENCES}

\section{C}

$\mathrm{D}$

Fig. 5 SEM photographs of HAp porous materials. C: Sintered body prepared by using $\alpha$ TCP cement and polymer beads, D: HAp crystals formed on sintered $\alpha \mathrm{TCP}$ ceramics,

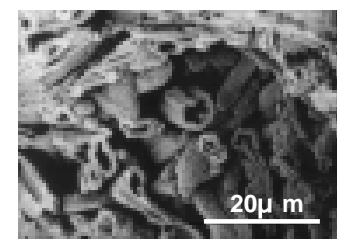

E

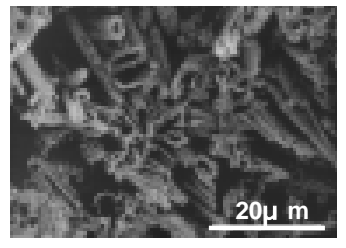

F
Fig. 6 SEM photographs of HAp porous materials. E and F: Tubular HAp and $\beta$ TCP prepared by using gypsum hardened body $^{16)}$.

\section{CLOSING}

The chemistry and characteristics of cement of the system $\mathrm{CaO}-\mathrm{P}_{2} \mathrm{O}_{5}$ have been described briefly. The systems $\mathrm{CaO}-\mathrm{Al}_{2} \mathrm{O}_{3}$ and $\mathrm{CaO}-\mathrm{SiO}_{2}$ are well- known for the base of Portland cement, and $\mathrm{CaO}-\mathrm{SO}_{3}$ for gypsum. Namely, hydraulic cement materials consisting of $\mathrm{Ca}$ salts of ${ }_{13} \mathrm{Al},{ }_{14} \mathrm{Si},{ }_{15} \mathrm{P}$ and ${ }_{16} \mathrm{~S}$ have just lined up. Furthermore various studies on the newly coming $\mathrm{CaO}-\mathrm{P}_{2} \mathrm{O}_{5}$ cement are desired to be going on.
1. H.Monma, T.Kanazawa, J. Ceram. Soc. Japan. , 84(4), 209-213(1976); 108(8), S75-S80(2000).

2. W.E.Brown, L.C.Chow, J. Dent. Res., 62(Spec. Issue), 672(1983); U.S.Pat. 4518430(1985); L.C.Chow, Adv. Dent. Res., 2(1), 181-186(1988).

3. H.Monma, Biomaterials ,15(1), 24-30 (1997).

4. W.E.Brown, L.C.Chow, Cements Res. Progress, 351-379(1986).

5. H.Monma ,A.Makishima ,M.Mitomo , Y.Ikegami, J. Ceram. Soc. Japan. ,96(8), 878-880(1988).

6. H.Monma, FC Report, 6(12), 475-480 (1988).

7. H.Monma, Y.Goto, T.Kohmura, J. Soc. Inorg. Mater. Japan, No.188, 11-16 (1984).

8. C.Durucan, P.W.Brown, J. Mater. Sci.,37 (5), 963-969(2002).

9. H.Monma, Kogyo Zairyo, 28(10), 90-102 (1980).

10. H.Takeuchi, J. Soc. Inorg. Mater. Japan, No.240, 52-59(1992).

11. M.Hirano, New Ceramics ,No.5 ,55-59 (1993).

12. H.Takeuchi, Spin \& Spinal Cord, 15(11), 1056-1063(2002).

13. O.Bermudez, J.A.Boltung, F.C.M. Driessens, J. Mater. Sci.:Mater. Med., 5 (3), 144-146(1994).

14. H.Monma, Zairyo Gijutsu ,13(5), 143147(1995).

15. J.A.Delgado, I.Harr, A.Almirall, S.del Valle, J.A.Planell, M.P.Ginebra :Key Eng. Mater. (Bioceramics 17), 284-286, 157 160(2005).

16. H.Monma, Y.Moriyoshi, T.Urano, T. Okura, S.Takahashi, Phos. Res. Bull., 19, 166-171(2005). 